\title{
Editorial
}

\section{Artificial turf: safe or out on ball fields around the world}

\author{
Journal of Exposure Science and Environmental Epidemiology (2008) 18, 533-534; doi:10.1038/jes.2008.56
}

The use of artificial turf or grass on athletic and recreational fields has increased over the past 40 years because of its durability and low maintenance. However, recent reports about lead being present in the fibers and then released from the fibers, potentially exposing children playing on these artificial turf surfaces have raised concerns. Furthermore, there are many who are also questioning the use of turf fields designed not only with synthetic fibers but also with a crumb rubber "infill".

High lead levels have been found by state and local agencies and other organizations in a number of "in-use" fields of varying ages and types of materials. The lead is mixed within the fibers as a lead chromate paint for bright coloration of the surface. It seems, however, that the surfaces degrade over time because of use and weathering. As a result, lead can be released from the older turf material by just wiping the surface with synthetic sweat or extracting from the turf fibers using a synthesis digestive system. In some cases, the amount on wipe exceeds residential guidelines for lead on floor surfaces, that is $40 \mu \mathrm{g} / \mathrm{ft}^{2}$, and it is likely that continued aging of the turf will result in even greater releases. Furthermore, the ease of mobilization would enhance the probability of re-suspension of lead into the breathing zone of athletes and other users, especially young children. Unfortunately, neither of these issues has been adequately examined for impact on the cumulative exposure and risk to the users even though these turfs have been sold for many years.

This past spring, for the first time, the Centers for Disease Control and Prevention recommended that older fields made of nylon should be tested for lead. Occasional use of these fields is not expected to present a major health hazard, although it is a good hygienic policy to wash the hands after playing on the fields and certainly before eating, as would be the advice whenever playing outdoors. However, it is not realistic to assume that this policy will always be followed and monitored. Thus, if high lead concentrations are measured on wipe samples collected from fields or the concentration of lead in the artificial turf is thousands of parts per million, then replacement of the field should be planned with interim use following the CDC guidance. This sensible guidance was recently "thrown under the bus" by the Consumer Product Safety Commission, which issued an advisory on 30 July 2008 that stated "CPSC Staff finds Synthetic Fields OK to
Install and OK to Play On." A review of the reasoning for their statement indicates that they made a hasty decision. Examples are (1) too few fields with lead-painted turf were studied as testing by a number of organizations indicates a range from 100 to over 11,000 p.p.m. of lead in fibers, (2) the CPSC evaluated only the incidental ingestion route, but overcorrected for contact and potential exposure, (3) they totally ignored the inhalation route of exposure, and (4) a contextual framework on the significance of lead exposure from leaded turf within the cumulative exposure to children was not considered. Recently, Congress overwhelmingly passed and President Bush signed a bill authorizing the CPSC to impose the toughest lead standards in the world, banning lead beyond minute levels in products for children 12 or younger age. Lead paint was a major factor in the recall of 45 million toys and children's items last year, including Cookie Monster and Thomas the Tank Engine toys. Many came from China. The CPSC action on synthetic turf should be consistent with this position.

In addition to the above, crumb rubber that is used as a fill on some of the above types of fields, and other fields that are made with turf that do NOT have lead in the turf fibers, presents a more complex issue. Neither systematic testing nor post-test evaluation has been performed on the composition and fate of either the Turf or the filler. Questions that need to be answered are the following: is the crumb rubber contaminated with metals as it comes from "ground up" used tires that have been in contact with many roadways and dirt surfaces; what is the surface temperature of the artificial turf as the crumb rubber is black and will absorb more heat than a grass surface; how are the fields safely disposed of once they exceed their usable lifetime; and what happens to the rubber material that does not stay attached to the turf as it becomes mobilized and is released into the environment or becomes attached to the skin and clothing of the users. Numerous mothers have told us that this crumb rubber comes home with the child and is distributed around the house. Furthermore, there are now residential uses of turf with and without "in fill" marketed in many colors with unspecified coloring agents. Is the rubber and turf safe?

For now the State of New York says non-lead synthetic turf with infill is safe in its advisories, with a note that it will reevaluate upon the receipt of more data. However, it has 
recently come to our attention that some crumb rubber-based infill fields have lead paint in the synthetic turf. How does that change the equation for the safety of the infill? Does the lead leach on to the crumb rubber, and find its way home?

At the present time, we believe that the million dollar + expense to produce and install a synthetic field by communities and athletic facilities demands a much more thorough understanding of the environmental impacts, human exposure and health risk implications associated with all synthetic turf products available on the market. This calls for a comprehensive evaluation of artificial turf by exposure scientists, and others in environmental science and environmental health sciences. This should be developed with cooperation among the users, regulatory agencies and the manufacturers of the turf products. In the end, the goal would be a set of guidelines for product composition and design to ensure this well-liked and increasingly available product for athletic field and home use has a true clean bill of health. The evaluation should consider durability, full life cycle cost, heat retention, human exposure to contaminants, ecological impact, and aesthetic properties. Following a prudent scientific approach to understanding the potential exposure, ecological and health effects from the material used on these fields will allow us to continue to hear the sounds "play ball" on synthetic turf athletic surfaces.

PAUL J. LIOY AND CLIFFORD P. WEISEL Exposure Science Division, Environmental and Occupational Health Sciences Institute of Robert Wood Johnson Medical School - UMDNJ and Rutgers University, 170 Frelinghuysen Road, Piscataway, NJ, USA 